\title{
TMPRSS4: an emerging potential therapeutic target in cancer
}

\author{
A L de Aberasturi ${ }^{1}$ and A Calvo*,1 \\ ${ }^{1}$ Department of Histology and Pathology and Oncology Division, CIMA of the University of Navarra, Pio XII, 55, 31008 Pamplona, \\ Spain
}

Altered expression and activity of proteases is a key event in cancer, particularly in relation to invasion, modification of the extracellular matrix and metastasis. The transmembrane protease, serine 4 (TMPRSS4) is closely related to other cancer-associated proteases, such as hepsin, TMPRSS2 and matriptase. We review in this study up-to-date information about expression, role, regulation and clinical relevance of TMPRSS4 in cancer. Increased expression of this protease is associated with acquisition of epithelial to mesenchymal transition, invasion and metastasis in vivo. Signalling in cancer cells involves upregulation of integrin- $\alpha 5$ (ITG- $\alpha 5$ ) and urokinase-type plasminogen activator (UPA), downregulation of E-cadherin and activation of uPA enzymatic activity at the plasma membrane, as well as phosphorylation of FAK, Src, Akt and ERK1/2 intracellularly. Upregulation of miR-205 hinders the protumorigenic effects elicited by TMPRSS4 through restoration of E-cadherin levels and direct targeting of ITG- $\alpha 5$. High levels of TMPRSS4 have been found in several types of solid tumours in patients, and association with poor prognosis has been consistently described. On the basis of this information and the structural characteristics of this druggable protease, we suggest that TMPRSS4 could be a novel potential therapeutic target in solid tumours.

\section{TMPRSS4, A TRANSMEMBRANE SERINE PROTEASE}

Proteolysis is a regulatory mechanism mediated by specific hydrolysis of peptide bonds (Lopez-Otin and Overall, 2002). Due to this post-translational modification, the role of numerous proteins is controlled by proteases, which then modulate a plethora of cellular mechanisms, such as cell growth, apoptosis, protein secretion, phagocytosis, signal transduction and extracellular matrix turnover (Puente et al, 2005). In humans, more than $2 \%$ of the genes code for a complex system of more than 700 proteases and inhibitors of proteases. The dysregulation of protease activity is related to different pathologies, including arthritis, cancer and neurogenerative and cardiovascular diseases (Puente et al, 2005).

On the basis of their mechanisms of catalysis, proteases are classified into serine, aspartyl, metallo, threonine and cysteine proteases (Puente et al, 2005). This large family of proteins can be found extracellularly, at the cellular surface, in the cytoplasm or within specific subcellular structures such as lysosomes. Some serine proteases exhibit a transmembrane domain through which they get anchored to the plasma membrane. Cell surface proteolysis has emerged as an important mechanism for the generation of biologically active factors that mediate a diverse range of cellular functions. Depending on the structure of the transmembrane domain, these serine proteases can be classified into three groups: type I (with a carboxy-terminal transmembrane domain), type II or TTSP (with an amino-terminal transmembrane domain spanning through the cytosol) and GPI (bound to the membrane by glycosyl-phosphatidylinositol; Netzel-Arnett et al, 2003). The type II family of serine proteases includes 20 members that are subdivided into four subfamilies: matriptase, hepsin/ transmembrane protease/serine (TMPRSS), HAT/differentially expressed in squamous cell carcinoma (DESC) and corin (Szabo and Bugge, 2008). In this review, we summarise the state of the art about the expression, role, signalling and clinical relevance of TMPRSS4 in cancer, where the importance of this membranebound serine protease is beginning to be acknowledged.

\section{TMPRSS4 STRUCTURE AND CATALYTIC ACTIVITY}

The transmembrane protease, serine 4 (TMPRSS4), previously referred to as TMPRSS3 (Wallrapp et al, 2000), is localised in the

*Correspondence: Dr A Calvo; E-mail: acalvo@unav.es

Received 14 April 2014; revised 17 June 2014; accepted 23 June 2014; published online 9 September 2014 
long arm of chromosome 11 (11q23.3). This gene contains $48,597 \mathrm{bp}$ and consists of 13 exons and 12 introns. Eighteen different transcripts of this gene can be generated, 2 of which are degraded due to nonsense-mediated decay and 8 of which do not give rise to a protein product. Of the remaining eight, three have incomplete coding sequence (CDS) in $5^{\prime}$ or $3^{\prime}$ and do not have either start or stop codon sequences. Accordingly, the TMPRSS4 gene codes for five isoforms with complete CDS. The canonic protein (TMPRSS4-1) is composed of 437 amino acids (with a predictive size of $48 \mathrm{kDa}$ and two glycosylation sites at 130 and 178 amino acids), whereas isoforms 2 and 3 differ in two and five amino acids, respectively (http://www.ensembl.org/index.html).

TMPRSS4 shares the following domains with the other TTSP family members: proteolytic, stem, transmembrane and cytoplasmic domains (Figure 1; Hooper et al, 2001). The proteolytic domain is highly conserved between different TTSPs and its activity is dependent on the presence of a 'catalytic triad' that includes the amino acids His, Asp and Ser. Enzymatic activity is also modulated by a substrate-binding pocket that determines the enzyme's specificity (Antalis et al, 2010). The stem region may include different regulatory and/or binding domains, as is the case for one LDL receptor class A domain present in TMPRSS4. In other TTSPs, this domain binds $\mathrm{Ca}^{2+}$ ions and has a role in the internalisation of macromolecules (Daly et al, 1995). The scavenger receptor domain is also present in the stem region. This domain is involved in binding of lipoproteins, lipids and polysaccharides (Netzel-Arnett et al, 2003). The remaining domains consist of the transmembrane region and a short cytoplasmic tail whose putative biological function in terms of cell signalling or cytoskeletal attachment is at present unknown (Hooper et al, 2001; Netzel-Arnett et al, 2003).

It is thought that all TTSPs are synthesised as zymogens that would need to be activated by proteolytic cleavage in a highly conserved motif that precedes the catalytic domain. On cleavage, it is likely that a disulphide bond maintains the catalytic domain linked to the rest of the protein and, therefore, anchored to the membrane (Hooper et al, 2001; Netzel-Arnett et al, 2003). Many TTSPs have been shown to be activated by autocatalysis, including TMPRSS2, matriptase, hepsin and TMPRSS4 (Szabo and Bugge, 2008). Other proteases can also activate pro-TTSP zymogens; activation by enterokinase has been suggested for TMPRSS4 (Min et al, 2014). Nonetheless, current knowledge about TMPRSS4 processing needs to be better substantiated.

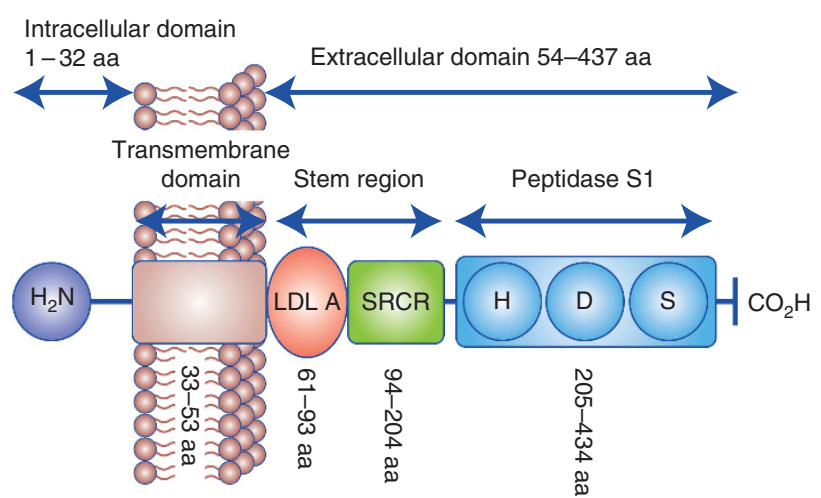

Figure 1. Schematic diagram of the TMPRSS4 structure. TMPRSS4 is a single-pass type II membrane protein. It contains a serine protease domain at the $\mathrm{C}$ terminus (peptidase S1), followed by a scavenger receptor cysteine-rich domain (SRDR) and a low-density lipoprotein receptor class $A$ domain. $H, D$ and $S$ in the serine protease domain indicate the position of the three catalytic residues histidine, aspartate and serine, respectively.
The existence of soluble forms of TTSPs has been described for HAT, enteropeptidase, matriptase and TMPRSS13 (MSPS), which implies that fragments of these proteases could be secreted (Hooper et al, 2001; Kim et al, 2001). The active TMPRSS4 protease domain can be released from cells in culture and are found in the conditioned medium (Min et al, 2014). This interesting finding opens the possibility that soluble fragments could be detected in serum of tumour-bearing patients and, therefore, that TMPRSS 4 can be used as a non-invasive diagnostic marker.

Although the specific substrates of TMPRSS4 are still underexplored, three proteins have been identified so far: (a) hemagglutinin of the influenza virus, which is necessary for virus infection (Bertram et al, 2010); (b) the urokinase-type plasminogen activator (uPA), whose activation enhances cancer cell invasion (see below in the next section); and (c) the epithelial sodium channel, on cleavage of the $\gamma$ subunit (Passero et al, 2012).

\section{ROLE OF TMPRSS4 AND ACTIVATION OF} INTRACELLULAR PATHWAYS IN CANCER

Among other proteases with clinical potential in cancer, such as uPA, matriptase, furin or stromelysin, TMPRSS4 could emerge as a new potential candidate. TMPRSS4 has been involved mainly in two functions at present: embryo development and cancer. In zebrafish embryos, this protease is necessary for organogenesis, as TMPRSS4 knockdown using morpholinos resulted in severe defects in tissue development and cell differentiation, including a disturbed skeletal muscle formation, a decelerated heartbeat and a degenerated vascular system (Ohler and Becker-Pauly, 2011). This result suggests that TMPRSS4 may modulate the activity of adhesion molecules involved in organ development (Ohler and Becker-Pauly, 2011). Generation of knockout and transgenic mice (lacking at this moment) would allow studying the involvement of this protease in healthy and pathological conditions, in a more relevant way for human diseases.

Most data about TMPRSS4 come from cancer development and metastasis studies. Its overexpression in tumours has been reported in pancreatic (Wallrapp et al, 2000), ovarian (Takahashi et al, 2013), thyroid (Ohler and Becker-Pauly, 2011), colorectal (Jung et al, 2008; Kim et al, 2010), lung (Larzabal et al, 2011; Figure 2), breast (Cheng et al, 2013b; Liang et al, 2013), cervical (Cheng et al, 2013b), gallbladder (Wu et al, 2014), gastric (Luo et al, 2013; Sheng et al, 2014) and liver cancer (Li et al, 2011).

Whether increased expression in cancer may be due to gene amplification, chromosome rearrangements, transcriptional

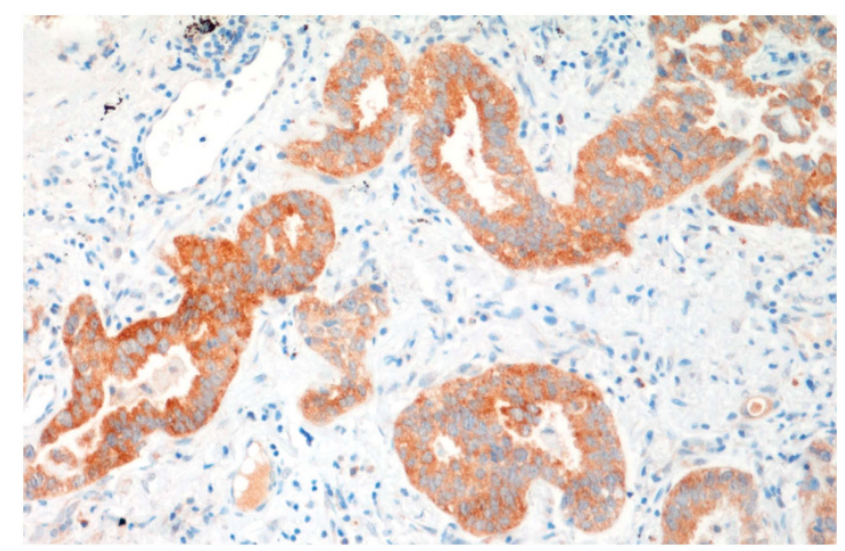

Figure 2. Immunohistochemical staining of a lung cancer specimen to localize TMPRSS4. 
dysregulation or other mechanisms is still unknown. In normal liver, TMPRSS4 and TMPRSS13 promoters have been shown to be methylated, whereas in hepatocellular carcinoma these genes are hypomethylated (Stefanska et al, 2011). These data suggest that a possible epigenetic dysregulation could be partially responsible for the increased expression observed in cancer. Nonetheless, these preliminary findings need further validation and expansion to other tumour types. TMPRSS4 has been shown to increase in hepatocarcinoma cells 30 days after irradiation, when expression of a first wave of VEGF- and MMP-9-induced cell response genes returns to normal levels ( $\mathrm{Li}$ et al, 2011). Overexpression of TMPRSS4 in the second wave of long-term response was critical for cell dissemination and metastasis of these cells (Li et al, 2011).

Several colon, lung and breast cancer cell lines express TMPRSS4 and have been used as in vitro models to uncover the role and signalling of TMPRSS4 (Jung et al, 2008; Larzabal et al, 2011). Migration and invasion are hallmarks of TMPRSS4 function in cancer cells. In lung and colon cancer, inhibition of this protease reduces migration (Jung et al, 2008; Larzabal et al, 2011) and invasion through matrigel, collagen type I and fibronectin-1 (Jung et al, 2008). Conversely, TMPRSS4 overexpression enhances migration and invasion in colon cancer (Jung et al, 2008). We have also reported an inhibition in the proliferation rates of lung cancer cell lines transfected with TMPRSS4-specific shRNA (Larzabal et al, 2011).

TMPRSS4 is responsible for the acquisition of an epithelial to mesenchymal transition (EMT) phenotype as well (Jung et al, 2008; Larzabal et al, 2011). In colon cancer cells, its overexpression leads to an intracellular signalling cascade that involves FAK, ERK1/2, Akt, Src and Racl activation (Kim et al, 2010; Figure 3). FAK and Rac1 signalling (which induces lapellipodia formation) are required for TMPRSS4-mediated invasion, changes in cell morphology and EMT (Kim et al, 2010). This pathway activates the transcription factors SIP1/ZEB2 and promotes E-cadherin loss (Jung et al, 2008; Li et al, 2011), a key event involved in EMT (Kim et al, 2010; Larzabal et al, 2011). In TMPRSS4-overexpressing cells, inhibition of PI3K or Src with specific compounds reduces invasiveness and causes actin reorganisation without restoration of E-cadherin expression.

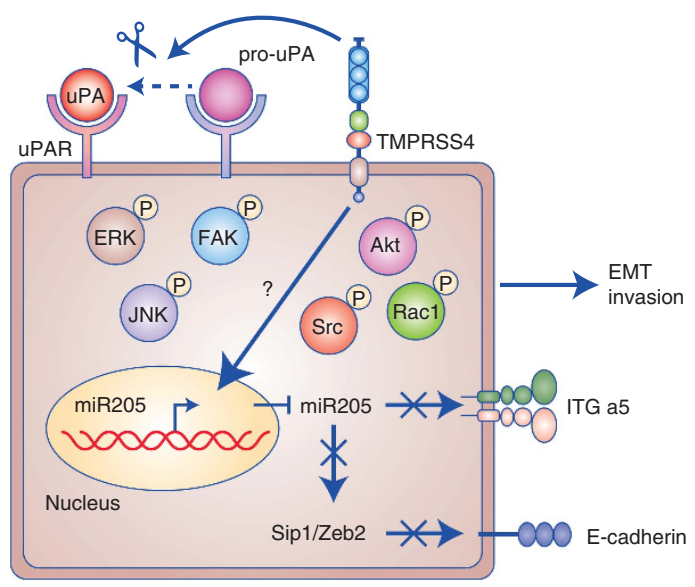

Figure 3. Scheme of molecules that participate in TMPRSS4-mediated signaling. Intracellular mediators include phosphorylated ERK, JNK, Akt, Src, FAK and Rac1. miR-205 targets integrin- $\alpha 5$ (ITG-a5), which is responsible for TMPRSS4-mediated invasiveness and EMT. miR-205 also targets Sip1/Zeb2, a repressor of E-cadherin. At the plasma membrane, TMPRSS4 cleaves the inactive form of uPA (pro-uPA) to accelerate invasiveness.
In addition, TMPRSS4 upregulates integrin- $\alpha 5$ (ITG- $\alpha 5)$ to induce invasiveness (Kim et al, 2010).

To identify new molecular mechanisms elicited by TMPRSS4, our group conducted transcriptomic profiling of TMPRSS4 knocked down lung cancer cells. MIR205HG, the gene coding for miR-205, a micro-RNA that suppresses metastasis (Iorio et al, 2009), was found to be overexpressed on TMPRSS4 downregulation (Larzabal et al, 2014). Increased levels of miR-205 impaired significantly cell growth (causing a $G_{0} / G_{1}$ cell cycle arrest), migration and attachment to fibronectin-1, and produced tumour shrinkage; moreover, we demonstrated that ITG- $\alpha 5$ is a new direct target of miR-205 in NSCLC and proposed a novel regulatory pathway involving TMPRSS4/miR-205/ITG- $\alpha 5$ (Larzabal et al, 2014). Therefore, data from both colon and lung cancer studies suggest a close relationship between TMPRSS4 and ITG- $\alpha 5$. Of note, blocking ITG- $\alpha 5$ antibodies (volociximab) are being evaluated in clinical trials for cancer treatment. The possibility of co-targeting both proteins could be an interesting approach to assess synergistic anti-tumour efficacy.

TMPRSS4 regulates uPA by a dual mechanism through increased gene expression and processing of pro-uPA into its active form (Min et al, 2014), which leads to enhanced invasion. uPA overexpression was mediated by JNK and transcription factors Sp1, Sp3 and AP-1. Moreover, immunohistochemical studies have shown co-expression of both proteases in human lung and prostate cancer tissues (Min et al, 2014). Therefore, it is possible that TMPRSS4 and UPA cooperate in tumours to accelerate metastasis.

Because of all these protumorigenic effects, TMPRSS4 has been suggested as a potential therapeutic target. Screening of a library of compounds against TMPRSS4 serine proteas activity identified several classes of inhibitory compounds, in particular a novel series of 2-hydroxydiarylamide derivatives (Kang et al, 2013). The leader compounds exhibited a relatively decent $\mathrm{IC}_{50}$ $(6-12 \mu \mathrm{M})$, but invasion assays in matrigel using TMPRSS4overexpressing SW480 cells revealed a modest inhibitory effect. Therefore, new families of more effective compounds should be developed and tested in different in vitro and in vivo models to support the translation of anti-TMPRSS4 therapy in clinical settings.

\section{PROGNOSTIC VALUE OF TMPRSS4}

Preclinical data showing increased malignancy in cells with high TMPRSS4 expression are in keeping with studies in human subjects. As mentioned in the previous section, TMPRSS4 is overexpressed in many solid tumours; importantly, this expression has been associated with poor outcome. In colorectal cancer, high TMPRSS4 protein levels were significantly correlated with advanced TNM stage and predicted shorter overall survival (OS) and disease-free survival (DFS). In Cox regression analysis, TMPRSS4 was an independent predictive factor of both OS and DFS (Huang et al, 2013). Similar results have been reported in patients with salivary adenoid cystic carcinoma (Dai et al, 2013). Analysis by western blot revealed a $>30$-fold increase in TMPRSS4 levels in these tumour tissues compared with matched noncancerous tissues. In this study, TMPRSS4 levels also correlated with TNM stage, as well as lymph node and distant metastasis. Multivariate analysis revealed that TMPRSS4 was an independent predictor of both OS and DFS.

In breast cancer, two independent studies have demonstrated the prognostic value of TMPRSS4 (Cheng et al, 2013a; Liang et al, 2013). In a series of 109 patients, protein levels of TMPRSS4 in tumours were significantly higher than those of non-malignant tissues. High expression of this protease correlated with lymph 
node metastasis, histopathological grade and tumour size $(>2 \mathrm{~cm})$, but not with oestrogen, progesterone or HER2 receptors. In univariate and multivariate analysis, high TMPRSS4 levels were associated with both DFS and OS (Liang et al, 2013). The other study found that high TMPRSS4 levels were observed in $62.4 \%$ breast cancer tissues (from a total number of 181 patients). The frequency of tumours with high TMPRSS4 expression was significantly higher in triple-negative breast cancer patients $(73.2 \%)$ than in non-triple-negative patients. They also confirmed correlation between high TMPRSS4 expression and lymph node metastasis and tumour size. Association between TMPRSS4 and reduced DFS and OS was found for both triple-negative and nontriple-negative tumours.

In keeping with in vitro studies that evidenced activation of ERK1/2 as a result of TMPRSS4 signalling, expression of both proteins in clinical samples from gastric cancer patients $(n=436)$ was found to be statistically correlated (Luo et al, 2013). High levels of TMPRSS4 were also associated with tumour size, lymph node and distal metastases, and TNM stage. Further multivariate analysis in these patients demonstrated that expression of TMPRSS4 was an independent prognostic factor. In a different study, TMPRSS4 was confirmed as an indicator of poor prognosis in gastric cancer (Sheng et al, 2014). The prognostic value of TMPRSS4 has also been recently shown in gallbladder cancer as well (Wu et al, 2014).

\section{CONCLUSION}

Increasing data on the role of TMPRSS4 in cancer development and metastasis suggest that this membrane-anchored serine protease merits further consideration as a novel potential therapeutic target in solid tumours. Although data in the literature on TMPRSS4 are still scarce, several lines of evidence support this statement: (a) TMPRSS4 has been proved to promote metastasis in preclinical models; (b) overexpression has been found in a variety of cancer types compared with normal tissues; (c) high levels are consistently associated with reduced DFS and OS; (d) it is a membrane-bound protein, which makes it an attractive target for the development of blocking antibodies or other biological inhibitory tools; and (e) TMPRSS4 extracellular fragments are shed into conditioned media of cultured cells, suggesting that they could be potentially found in serum samples from patients. We conclude that TMPRSS4 is an emerging potential cancer target, although future studies should determine important mechanistic and translational aspects of this protease before being considered as a consolidated target.

\section{ACKNOWLEDGEMENTS}

We are thankful to RTICC (10/00166) and FIS (PI13/00093) for funding this study.

\section{REFERENCES}

Antalis TM, Buzza MS, Hodge KM, Hooper JD, Netzel-Arnett S (2010) The cutting edge: membrane-anchored serine protease activities in the pericellular microenvironment. Biochem J 428(3): 325-346.

Bertram S, Glowacka I, Steffen I, Kuhl A, Pohlmann S (2010) Novel insights into proteolytic cleavage of influenza virus hemagglutinin. Rev Med Virol 20(5): 298-310.

Cheng D, Kong H, Li Y (2013a) TMPRSS4 as a poor prognostic factor for triple-negative breast cancer. Int J Mol Sci 14(7): 14659-14668.

Cheng D, Liang B, Li Y (2013b) High TMPRSS4 expression is a predictor of poor prognosis in cervical squamous cell carcinoma. Cancer Epidemiol 37(6): 993-997.
Dai W, Zhou Q, Xu Z, Zhang E (2013) Expression of TMPRSS4 in patients with salivary adenoid cystic carcinoma: correlation with clinicopathological features and prognosis. Med Oncol 30(4): 749.

Daly NL, Scanlon MJ, Djordjevic JT, Kroon PA, Smith R (1995) Three-dimensional structure of a cysteine-rich repeat from the low-density lipoprotein receptor. Proc Natl Acad Sci USA 92(14): 6334-6338.

Hooper JD, Clements JA, Quigley JP, Antalis TM (2001) Type II transmembrane serine proteases. Insights into an emerging class of cell surface proteolytic enzymes. J Biol Chem 276(2): 857-860.

Huang A, Zhou H, Zhao H, Quan Y, Feng B, Zheng M (2013) High expression level of TMPRSS4 predicts adverse outcomes of colorectal cancer patients. Med Oncol 30(4): 712.

Iorio MV, Casalini P, Piovan C, Di Leva G, Merlo A, Triulzi T, Menard S, Croce CM, Tagliabue E (2009) microRNA-205 regulates HER3 in human breast cancer. Cancer Res 69(6): 2195-2200.

Jung H, Lee KP, Park SJ, Park JH, Jang YS, Choi SY, Jung JG, Jo K, Park DY, Yoon JH, Park JH, Lim DS, Hong GR, Choi C, Park YK, Lee JW, Hong HJ, Kim S, Park YW (2008) TMPRSS4 promotes invasion, migration and metastasis of human tumor cells by facilitating an epithelial-mesenchymal transition. Oncogene 27(18): 2635-2647.

Kang S, Min HJ, Kang MS, Jung MG, Kim S (2013) Discovery of novel 2-hydroxydiarylamide derivatives as TMPRSS4 inhibitors. Bioorg Med Chem Lett 23(6): 1748-1751.

Kim DR, Sharmin S, Inoue M, Kido H (2001) Cloning and expression of novel mosaic serine proteases with and without a transmembrane domain from human lung. Biochim Biophys Acta 1518(1-2): 204-209.

Kim S, Kang HY, Nam EH, Choi MS, Zhao XF, Hong CS, Lee JW, Lee JH, Park YK (2010) TMPRSS4 induces invasion and epithelial-mesenchymal transition through upregulation of integrin alpha5 and its signaling pathways. Carcinogenesis 31(4): 597-606.

Larzabal L, de Aberasturi AL, Redrado M, Rueda P, Rodriguez MJ, Bodegas ME, Montuenga LM, Calvo A (2014) TMPRSS4 regulates levels of integrin alpha5 in NSCLC through miR-205 activity to promote metastasis. Br J Cancer 110(3): 764-774.

Larzabal L, Nguewa PA, Pio R, Blanco D, Sanchez B, Rodriguez MJ, Pajares MJ, Catena R, Montuenga LM, Calvo A (2011) Overexpression of TMPRSS4 in non-small cell lung cancer is associated with poor prognosis in patients with squamous histology. Br J Cancer 105(10): 1608-1614.

Li T, Zeng ZC, Wang L, Qiu SJ, Zhou JW, Zhi XT, Yu HH, Tang ZY (2011) Radiation enhances long-term metastasis potential of residual hepatocellular carcinoma in nude mice through TMPRSS4-induced epithelial-mesenchymal transition. Cancer Gene Ther 18(9): 617-626.

Liang B, Wu M, Bu Y, Zhao A, Xie F (2013) Prognostic value of TMPRSS4 expression in patients with breast cancer. Med Oncol 30(2): 497.

Lopez-Otin C, Overall CM (2002) Protease degradomics: a new challenge for proteomics. Nat Rev Mol Cell Biol 3(7): 509-519.

Luo ZY, Wang YY, Zhao ZS, Li B, Chen JF (2013) The expression of TMPRSS4 and Erk1 correlates with metastasis and poor prognosis in Chinese patients with gastric cancer. PLoS One 8(7): e70311.

Min HJ, Lee MK, Lee JW, Kim S (2014) TMPRSS4 induces cancer cell invasion through pro-uPA processing. Biochem Biophys Res Commun 446(1): 1-7.

Netzel-Arnett S, Hooper JD, Szabo R, Madison EL, Quigley JP, Bugge TH, Antalis TM (2003) Membrane anchored serine proteases: a rapidly expanding group of cell surface proteolytic enzymes with potential roles in cancer. Cancer Metastasis Rev 22(2-3): 237-258.

Ohler A, Becker-Pauly C (2011) Morpholino knockdown of the ubiquitously expressed transmembrane serine protease TMPRSS4a in zebrafish embryos exhibits severe defects in organogenesis and cell adhesion. Biol Chem 392(7): 653-664.

Passero CJ, Mueller GM, Myerburg MM, Carattino MD, Hughey RP, Kleyman TR (2012) TMPRSS4-dependent activation of the epithelial sodium channel requires cleavage of the gamma-subunit distal to the furin cleavage site. Am J Physiol Renal Physiol 302(1): F1-F8.

Puente XS, Sanchez LM, Gutierrez-Fernandez A, Velasco G, Lopez-Otin C (2005) A genomic view of the complexity of mammalian proteolytic systems. Biochem Soc Trans 33(Pt 2): 331-334.

Sheng H, Shen W, Zeng J, Xi L, Deng L (2014) Prognostic significance of TMPRSS4 in gastric cancer. Neoplasma 61(2): 213-217.

Stefanska B, Huang J, Bhattacharyya B, Suderman M, Hallett M, Han ZG, Szyf M (2011) Definition of the landscape of promoter DNA hypomethylation in liver cancer. Cancer Res 71(17): 5891-5903. 
Szabo R, Bugge TH (2008) Type II transmembrane serine proteases in development and disease. Int J Biochem Cell Biol 40(6-7): 1297-1316.

Takahashi T, Uehara H, Izumi K (2013) Inhibitory effect of soluble EP2 receptor on ovarian tumor growth in nude mice and utility of TMPRSS4 as a combinatorial molecular target. Int J Oncol 43(2): 416-424.

Wallrapp C, Hahnel S, Muller-Pillasch F, Burghardt B, Iwamura T,

Ruthenburger M, Lerch MM, Adler G, Gress TM (2000) A novel transmembrane serine protease (TMPRSS3) overexpressed in pancreatic cancer. Cancer Res 60(10): 2602-2606.
Wu XY, Zhang L, Zhang KM, Zhang MH, Ruan TY, Liu CY, Xu JY (2014) Clinical implication of TMPRSS4 expression in human gallbladder cancer. Tumour Biol 35(6): 5481-5486.

(c) (i) (2) This work is licensed under the Creative Commons Attribution-NonCommercial-Share Alike 3.0 Unported License. To view a copy of this license, visit http://creativecommons. org/licenses/by-nc-sa/3.0/ 